\title{
TWO-DIMENSIONAL, ALMOST PERIODIC LINEAR SYSTEMS WITH PROXIMAL AND RECURRENT BEHAVIOR
}

\author{
RUSSELL A. JOHNSON
}

\begin{abstract}
We prove that there exist two-dimensional, almost periodic linear systems, with arbitrary basis of frequencies, the angular coordinates of whose solutions have both proximal and recurrent behavior. Such behavior is completely unlike that of any periodic system.
\end{abstract}

1. Introduction. Consider a two-dimensional, linear differential equation

$$
\dot{x}=A(t) x, \quad \operatorname{trace} A(t) \equiv 0,
$$

where $A(t)$ is periodic. If $x_{1}(t)$ and $x_{2}(t)$ are solutions of $(*)$, let $\Theta\left(x_{1}(t), x_{2}(t)\right)$ denote the angular separation between them. Say $x_{1}$ and $x_{2}$ are proximal if inf $\Theta\left(x_{1}(t), x_{2}(t)\right)=0$. If every pair $x_{1}(t), x_{2}(t)$ of solutions of (*) is proximal, then a periodic change of variables $x=p(t) y$ takes (*) to the form

$$
\dot{y}=\left(\begin{array}{ll}
0 & 0 \\
1 & 0
\end{array}\right) y .
$$

It follows that (*) has a periodic solution $x_{0}(t)$ such that $\lim _{|t| \rightarrow \infty} \Theta\left(x(t), x_{0}(t)\right)=0$ for every solution $x(t)$.

Now suppose $A(t)$ is almost periodic. Suppose inf $\Theta\left(x_{1}(t), x_{2}(t)\right)=0$ for each pair of solutions $x_{1}(t), x_{2}(t)$ of $(*)$. We look for analogies with the periodic case. For example, let $(\Sigma, \mathbf{R})$ be the projective flow (2.3) generated by (*). We ask if $(\Sigma, \mathbf{R})$ admits a minimal, almost automorphic subflow $(M, \mathbf{R})(2.1$; see [13]). Such an $M$ would be an analogue of the periodic solution $x_{0}(t)$ mentioned in the last paragraph.

We will show that the answer to this question is "no". We will show that there are equations (*) whose projective flow is a minimal, proximal extension of the hull [11] of $A$ (see 2.1). This means that:

(i) inf, $\Theta\left(x_{1}(t), x_{2}(t)\right)=0$ for all solutions $x_{1}(t), x_{2}(t)$ of $(*)$;

(ii) the angular coordinate $\theta(t)$ of every solution $x(t)$ of (*) wanders densely through the set of all directions in the sense that, if $\theta_{0} \in[0,2 \pi)$, then there is a sequence $t_{n} \rightarrow \infty$ such that $A\left(t+t_{n}\right) \rightarrow A(t)$ uniformly, and $\theta\left(t_{n}\right) \rightarrow \theta_{0} \bmod 2 \pi$. We will also find equations (*) such that, in addition to the above, $(\Sigma, \mathbf{R})$ is strictly ergodic (2.1).

We will use ideas similar to those of Glasner and Weiss [6. They in turn refer to a paper of Anosov and Katov [1] and Fathi-Herman [4]. We note that Ellis (see [3])

Received by the editors June 17, 1980.

AMS (MOS) subject classifications (1970). Primary 34A30, 54H20.

(C) 1981 American Mathematical Society 0002-9939/81/0000-0322/\$02.50 
has developed a technique for producing examples like ours, and higherdimensional analogues. Our development is relatively concrete; also, our proofs are self-contained, and involve only the most simple constructions.

The author would like to thank Professor Robert Ellis for showing him the preprint by Glasner and Weiss [6].

\section{Preliminaries.}

2.1 Definitions. Let $X$ be a compact metric space. A (real) flow on $X$, denoted $(X, \mathbf{R})$, is given by a continuous map $\Phi: X \times \mathbf{R} \rightarrow X:(x, t) \rightarrow x \cdot t$ such that: (i) $x \cdot 0=x(x \in X)$; (ii) $x \cdot(t+s)=(x \cdot t) \cdot s \quad(x \in X ; t, s \in \mathbf{R})$. Say $(X, \mathbf{R})$ is minimal if every orbit $\{x \cdot t \mid t \in \mathbf{R}\}$ is dense in $X(x \in X)$. Say $(X, \mathbf{R})$ is uniquely ergodic if there is a unique R-invariant measure [10] on $X$; if, in addition, $(X, \mathbf{R})$ is minimal, then it is strictly ergodic. Say $(X, \mathbf{R})$ is almost periodic (a.p) if, given $\varepsilon>0$, there is a $\delta>0$ such that $d(x, y)<\delta$ implies $d(x \cdot t, y \cdot t)<\varepsilon(x \in X, y \in X, t \in$ $\mathbf{R})$. Here $d$ is some metric on $X$. If $(Y, \mathbf{R})$ is another flow with $Y$ compact metric, then $(X, \mathbf{R})$ is an extension of $(Y, \mathbf{R})$ if there is a continuous surjection $\pi: X \rightarrow Y$ such that $\pi(x \cdot t)=\pi(x) \cdot t \quad(x \in X, t \in \mathbf{R})$. In this situation, suppose inf,$d\left(x_{1} \cdot t, x_{2} \cdot t\right)=0$ whenever $\pi\left(x_{1}\right)=\pi\left(x_{2}\right)$; then $(X, \mathbf{R})$ is a proximal extension of $(Y, \mathbf{R})$. Also, if $(Y, \mathbf{R})$ is a.p. and minimal, and if $\operatorname{card} \pi^{-1}(y)=1$ for some $y \in Y$, then $(X, \mathbf{R})$ is almost automorphic [13].

2.2 Definitions. Let $\Omega$ be a compact metric space, and let $(\Omega, \mathbf{R})$ be an a.p. minimal flow. Let $L(2)$ be the set of $2 \times 2$, real matrices, with

$$
|B|=\sup \{|B x|:|x|=1\}
$$

here $|\cdot|$ is the Euclidean norm on $\mathbf{R}^{2}$. Let $a: \Omega \rightarrow L(2)$ be continuous, and consider the linear differential equations

$$
\dot{x}=a(\omega \cdot t) x \quad\left(x \in \mathbf{R}^{2}, \omega \in \Omega\right) .
$$

Every almost periodic ODE $\dot{x}=A(t) x$ induces an a.p. minimal flow $(\Omega, \mathbf{R})$ $(\Omega=\text { hull of } A \text { ), and a collection of equations (1) })_{\omega, a}[11]$. From now on, we fix some a.p. minimal flow $(\Omega, \mathbf{R})$.

2.3 Definitions. Equations (1) $)_{\omega, a}$ define a flow on $\Omega \times \mathbf{R}^{2}$ via $\left(\omega, x_{0}\right) \cdot t=$ $(\omega \cdot t, x(t))$, where $x(t)$ is the solution to $(1)_{\omega, a}$ such that $x(0)=x_{0}[11]$. Let $\mathbf{P}^{1}$ be real, one-dimensional projective space, and let $\Sigma=\Omega \times \mathbf{P}^{1}$. The flow $\left(\Omega \times \mathbf{R}^{2}, \mathbf{R}\right)$ induces a flow on $\Sigma$ via $(\omega, \mathfrak{l}) \cdot t=(\omega \cdot t, \mathfrak{I}(t))$. Here $\mathfrak{I}$ is a line through the origin in $\mathbf{R}^{2}$, and $\mathfrak{I}(t)$ its image after time $t$. Denote this flow by $\left(\Sigma_{a}, \mathbf{R}\right)$ to indicate the dependence on $a$. Then $\left(\Sigma_{a}, \mathbf{R}\right)$ describes the angular evolution of solutions of equations (1) $)_{\omega, a}$. It is the projective flow defined by equations $(1)_{\omega, a}$.

2.4 Definttion. Since $(\Omega, \mathbf{R})$ is a.p. minimal, it may be given the structure of a compact, abelian, topological group with dense subgroup $\mathbf{R}$ [2]. Let $\nu$ be normalized Haar measure on $\Omega$. Define

$$
C_{0}(\Omega)=\left\{b: \Omega \rightarrow \mathbf{R} \mid \int_{\Omega} b(\omega) d \nu(\omega)=0\right\}
$$


2.5 REMARKS. Let $b \in C_{0}(\omega)$, and consider the equations

$$
\dot{x}=\left(\begin{array}{cc}
0 & -b(\omega \cdot t) \\
b(\omega \cdot t) & 0
\end{array}\right) x \quad(\omega \in \Omega, t \in \mathbf{R}) .
$$

It is known that the flow on $\Sigma$ induced by equations $(2)_{\omega, b}$ is minimal if (and only if) $\int_{0}^{t} b(\omega \cdot s) d s$ is unbounded for some (hence any) $\omega \in \Omega$. It is strictly ergodic, if, in addition, the equation $r(\omega \cdot t)-r(\omega)=\int_{0}^{t} b(\omega \cdot s) d s(\omega \in \Omega, t \in \mathbf{R})$ has no $\nu$-measurable solution $r$ [5], [8].

\section{Results.}

3.1 Definitions. Let $P(\Omega)=\{p: \Omega \rightarrow L(2) \mid p$ is continuous, det $p(\omega)=1$ for all $\omega \in \Omega$, and $p^{\prime}: \Omega \rightarrow L(2):\left.\omega \rightarrow(d / d t) p(\omega \cdot t)\right|_{t=0}$ is continuous $\}$. Then, let

$$
\begin{array}{r}
S_{0}=\left\{a: \Omega \rightarrow L(2) \mid a(\omega)=p^{-1}(\omega)\left(\begin{array}{cc}
0 & -b(\omega) \\
b(\omega) & 0
\end{array}\right) p(\omega)\right. \\
\left.-p^{-1}(\omega) p^{\prime}(\omega) \text { for some } p \in P(\Omega) \text { and some } b \in C_{0}(\Omega)\right\} .
\end{array}
$$

3.2 REMARK. We can and will identify $S_{0}$ with the set of all collections (1) $)_{\omega, a}$ of equations that are obtained from a collection $(2)_{\omega, b}$ by a "strong Perron transformation" $x=p(\omega \cdot t) y(\omega \in \Omega)$, where $\operatorname{det} p(\omega) \equiv 1$.

3.3 Definitions. Let $\mathcal{C}=\{a: \Omega \rightarrow L(2) \mid a$ is continuous $\}$. Norm $C$ as follows: $\|a\|=\sup \{|a(\omega)|: \omega \in \Omega\}$; then $\mathcal{C}$ is a Banach space. Define $S=\operatorname{cls} S_{0} \subset \mathcal{C}$.

3.4 REMARK. If $a \in S$, then trace $a(\omega)=0(\omega \in \Omega)$.

3.5 Assumption. From now on, assume $(\Omega, \mathbf{R})$ is not periodic (i.e., $\Omega$ is not a circle). Recall $(\Omega, \mathbf{R})$ is a.p. minimal (2.2).

3.6 Proposition. There is a residual subset of $S_{1}$ of $S$ such that, if $a \in S_{1}$, then $\left(\Sigma_{a}, \mathbf{R}\right)$ is minimal.

Proof. Let $\left\{O_{n}: n \geqslant 1\right\}$ be a countable base for the topology of $\Sigma$. For each $n>1$, let $C_{n}=\left\{a \in S \mid\right.$ the flow $\left(\Sigma_{a}, \mathbf{R}\right)$ contains a minimal set which does not intersect $\left.O_{n}\right\}$. Then $C_{n}$ is closed in $S$. For, let $a_{m} \rightarrow a, a_{m} \in C_{n}$. Then $\left(\Sigma_{a_{m}}, \mathbf{R}\right)$ contains a minimal set $F_{m}$ such that $F_{m} \cap O_{n}=\varnothing(m>1)$. Perhaps choosing a subsequence, we may assume $F_{m} \rightarrow F \subset \Sigma$ in the Hausdorff metric on the space of closed subsets of $\Sigma$ [13]. Then $F$ is a compact invariant subset of $\left(\Sigma_{a}, \mathbf{R}\right)$, and $F \cap O_{n}=\varnothing$. Since $F$ contains a minimal subset, $C_{n}$ is closed.

Now, $\left\{b \in C_{0}(\Omega) \mid \int_{0}^{t} b(\omega \cdot s) d s\right.$ is unbounded for some $\left.\omega \in \Omega\right\}$ is dense in $C_{0}(\Omega)$, since $(\Omega, \mathbf{R})$ is not periodic. By 2.5 and the definition of $S,\left\{a \in S \mid\left(\Sigma_{a}, \mathbf{R}\right)\right.$ is minimal $\}$ is dense in $S$. Hence $S_{n}$ is nowhere dense in $S$, so $S_{1}=S \sim \cup_{n=1}^{\infty} S_{n}$ is a residual subset of $S$. Clearly $a \in S_{1} \Leftrightarrow\left(\Sigma_{a}, \mathbf{R}\right)$ is minimal.

3.7 Proposition. There is a residual subset $S_{2}$ of $S$ such that, if $a \in S_{2}$, then equation (1) $)_{\omega, a}$ admits an unbounded solution for some (hence all) $\omega \in \Omega$.

Proof. For each integer $N \geqslant 1$, let $C_{N}=\{a \in S \mid \text { every solution } x(t) \text { to (1) })_{\omega, a}$ such that $|x(0)|=1$ satisfies $|x(t)|<N(\omega \in \Omega, t \in \mathbf{R})\}$. Clearly $C_{N}$ is a closed 
subset of $S$. Define $S_{2}=S \sim \cup_{N=1}^{\infty} C_{N}$; then $S_{2}=\{a \in S \mid$ some equation (1) (a,a admits an unbounded solution $\}$.

To prove 3.7, we must show that each $C_{N}$ has dense complement in $S$. Using the definition of $S$ and the fact that $\operatorname{det} p(\omega)=1(\omega \in \Omega)$ for all $p \in P(\Omega)$, we see that it suffices to prove the following. Let $b \in C_{0}(\Omega), N>1$, and $\varepsilon>0$ be given. Then there is a $t_{0}=t_{0}(N, b, \varepsilon)$ in $\mathbf{R}$ and an $\omega_{0}=\omega_{0}(b)$ in $\Omega$ such that, for each $\theta \in \mathbf{R}$, the $\varepsilon$-neighborhood of $\left(\begin{array}{c}0-b \\ b\end{array}\right)$ in $S$ contains an element $a=a(\theta)$ such that equation $(1)_{\omega_{0} a}$ admits a solution $x(t)$ with $x\left(t_{0}\right) /\left|x\left(t_{0}\right)\right|=(\cos \theta, \sin \theta)$, and $\left|x\left(t_{0}\right)\right| /|x(0)|>$ $N$.

So, choose a function $b_{1} \in C_{0}(\Omega)$ such that $\sup \left\{\left|b(\omega)-b_{1}(\omega)\right|: \omega \in \Omega\right\}<\varepsilon / 2$, and $\int_{0}^{t} b_{1}(\omega \cdot s) d s$ is bounded for some (hence all) $\omega \in \Omega$. We may write

$$
\int_{0}^{t} b_{1}(\omega \cdot s) d s=B_{1}(\omega \cdot t)-B_{1}(\omega)
$$

where $B_{1} \in C_{0}(\Omega)$. Let

$$
p_{1}(\omega)=\left(\begin{array}{cc}
\cos B_{1}(\omega) & -\sin B_{1}(\omega) \\
\sin B_{1}(\omega) & \cos B_{1}(\omega)
\end{array}\right) \quad(\omega \in \Omega) .
$$

Note that the change of variables $x=p_{1}(\omega \cdot t) y$ takes equations $(2)_{\omega, b_{1}}$ to the form $\dot{y}=0$ for all $\omega \in \Omega$. Pick $\omega_{0} \in \Omega$ such that $B_{1}\left(\omega_{0}\right)=0$.

Next, choose a function $u \in C_{0}(\Omega)$ such that:

(i) $\sup \{\exp u(\omega) \mid \omega \in \Omega\}>N$;

(ii) if $u^{\prime}(\omega)=\left.(d / d t) u(\omega \cdot t)\right|_{t=0}$, then $\left|u^{\prime}(\omega)\right|<\varepsilon / 2(\omega \in \Omega)$;

(iii) $u\left(\omega_{0}\right)=0$.

We will see momentarily that such a $u$ may be found. Let

$$
p_{2}(\omega)=\left(\begin{array}{cc}
\exp -u(\omega) & 0 \\
0 & \exp u(\omega)
\end{array}\right) \quad(\omega \in \Omega) ;
$$

also let

$$
R_{\varphi}=\left(\begin{array}{cc}
\cos \varphi & -\sin \varphi \\
\sin \varphi & \cos \varphi
\end{array}\right) \text { for any } \varphi \in \mathbf{R} .
$$

Choose $t_{0}$ so that $\exp u\left(\omega_{0} \cdot t_{0}\right)>N$, and let

$$
p_{1}\left(\omega_{0} \cdot t_{0}\right)=\left(\begin{array}{cc}
\cos \bar{\theta} & -\sin \bar{\theta} \\
\sin \bar{\theta} & \cos \bar{\theta}
\end{array}\right) .
$$

It may now be checked that the change of variables

$$
x=p_{1}(\omega \cdot t) p_{2}(\omega \cdot t) R_{-\theta+\bar{\theta}} p_{1}^{-1}(\omega \cdot t) y
$$

takes equations $(2)_{\omega, b_{1}}$ to the form $\dot{y}=Q(\omega \cdot t) y$, where

$$
Q(\omega)=p_{1}(\omega) R_{\theta-\bar{\theta}} p_{2}^{-1}(\omega) p_{2}^{\prime}(\omega) R_{-\theta+\bar{\theta}} p_{1}^{-1}(\omega)-p_{1}(\omega)\left(p_{1}^{-1}\right)^{\prime}(\omega) \quad(\omega \in \Omega) .
$$

Since

$$
\left(\begin{array}{cc}
0 & -b_{1}(\omega) \\
b_{1}(\omega) & 0
\end{array}\right)=-p_{1}(\omega)\left(p_{1}^{-1}\right)^{\prime}(\omega)
$$


and since $\left\|p_{1} R_{\theta-\bar{\theta}} p_{2}^{-1} p_{2}^{\prime} R_{-\theta+\bar{\theta}} p_{1}^{-1}\right\|<\varepsilon / 2$ by choice of $u$, we see that

$$
\left|Q(\omega)-\left(\begin{array}{cc}
0 & -b(\omega) \\
b(\omega) & 0
\end{array}\right)\right|<\varepsilon \quad(\omega \in \Omega) .
$$

Also, if $y(t)$ satisfies $\dot{y}=Q(\omega \cdot t) y$, and $y(0)=(\cos (\theta-\bar{\theta}), \sin (\theta-\bar{\theta}))$, then $\left|y\left(t_{0}\right)\right|$ $>N$, and $y\left(t_{0}\right) /\left|y\left(t_{0}\right)\right|=(\cos \theta, \sin \theta)$.

Finally, we show that $u$ may be chosen in the indicated way. We proceed as in [7, §4]. Corresponding to $(\Omega, \mathbf{R})$ is some module of frequencies $\left\{\lambda_{K}\right\}_{K=1}^{\infty}$. Any Bohr almost periodic function $f: \mathbf{R} \rightarrow \mathbf{R}$ whose frequency module is contained in $\left\{\lambda_{K}\right\}$ may be viewed as a continuous function on $\Omega$, as follows. Let $\omega_{0} \in \Omega$ be as above, and define $\tilde{f}\left(\omega_{0} \cdot t\right)=f(t)(t \in \mathbf{R})$; then $\tilde{f}$ admits a unique continuous extension to S. Now let $\tilde{u}\left(\omega_{0} \cdot t\right)=[\ln (2 N)] \cdot \sin \lambda_{\mathrm{l}} t$, where $\lambda_{\mathrm{I}} \in\left\{\lambda_{K}\right\}$ is chosen so that $0 \neq\left|\lambda_{\mathrm{I}}\right|$ $<(\varepsilon / 2) \ln (2 N)$. This may be done because $(\Omega, \mathbf{R})$ is not periodic. Let $u$ be the unique continuous extension of $\tilde{u}$ to $\Omega$. Then $u \in C_{0}(\Omega)$. Also, $u^{\prime}$ is in $C_{0}(\Omega)$, and is the unique continuous extension to $\Omega$ of $\tilde{u}^{\prime}\left(\omega_{0} \cdot t\right)=-\lambda_{\mathrm{I}} \cdot[\ln (2 N)] \cdot \cos \lambda_{\mathrm{I}} t$. It is clear that $u$ satisfies the desired conditions.

3.8 Corollary. There is a residual subset $S_{3}$ of $S$ such that, if $a \in S_{3}$, then $\left(\Sigma_{a}, \mathbf{R}\right)$ is minimal, and is a proximal extension of $(\Omega, \mathbf{R})$.

Proof. Let $S_{3}=S_{1} \cap S_{2}$, where $S_{1}$ resp. $S_{2}$ is defined in 3.6 (resp. 3.7). If $a \in S_{3}$, then $\left(\Sigma_{a}, \mathbf{R}\right)$ is minimal, and some equation (1) $)_{\omega, a}$ admits an unbounded solution. If $\left(\Sigma_{a}, \mathbf{R}\right)$ is not a proximal extension of $(\Omega, \mathbf{R})$, then $[9, \S 7]$ implies that there is a minimal subflow $(M, \mathbf{R})$ of $\left(\Sigma_{a}, \mathbf{R}\right)$ such that $\operatorname{card}\left(M \cap\{\omega\} \times \mathbf{P}^{1}\right)=2$ for a residual set of $\omega \in \Omega$. Hence $M \neq \Sigma_{a}$. This contradicts minimality of $\left(\Sigma_{a}, \mathbf{R}\right)$; hence $\left(\Sigma_{a}, \mathbf{R}\right)$ is a proximal extension of $(\Omega, \mathbf{R})$.

3.9 Proposition. Let $S_{4}=\left\{a \in S \mid\left(\Sigma_{a}, \mathbf{R}\right)\right.$ is uniquely ergodic $\}$. Then $S_{4}$ is a residual subset of $S$.

Proof. From [7, §4], we see that there is a residual subset $C_{1} \subset C_{0}(\Omega)$ such that, if $b \in C_{1}$, then the equation $r(\omega \cdot t)-r(\omega)=\int_{0}^{t} b(\omega \cdot s) d s$ has no measurable solution. Hence, using 2.5 , we see that $S_{4}$ is dense in $S$.

We now proceed as in [6]. Let $\left\{f_{j}\right\}_{j=1}^{\infty}$ be a countable dense subset of $C(\Sigma)$ ( $=$ the usual space of continuous, real-valued functions on $\Sigma$ ). Given $\varepsilon>0$, let $V_{j, e}=\{a \in S \mid$ there exists a constant $\alpha=\alpha(j)$ and a "time" $t \neq 0$ such that $\left|(1 / t) \int_{0}^{t} f_{j}(\sigma \cdot s) d s-\alpha(j)\right|<\varepsilon$ for all $\left.\sigma \in \Sigma\right\}$; here $\sigma \cdot s$ is the "position" of $\sigma$ after time $s$ under the flow $\left(\Sigma_{a}, \mathbf{R}\right)$. Clearly $V_{j, e}$ is open in $S$. It is not hard to see that $\bigcap_{j=1}^{\infty} \cap_{n=1}^{\infty} V_{j, 1 / n}=S_{4}$. We conclude that $S_{4}$ is a residual subset of $S$.

Finally, taking the intersection of $S_{3}$ and $S_{4}$, we obtain

3.10 TheOREM. There is a residual subset $S_{5}$ of $S$ such that, if a $\in S_{5}$, then $\left(\Sigma_{a}, \mathbf{R}\right)$ is minimal, strictly ergodic, and is a proximal extension of $(\Omega, \mathbf{R})$.

\section{REFERENCES}

1. D. V. Anosov and A. B. Katok, New examples in smooth engodic theory. Engodic diffeomorphisms, Trudy Moscov. Mat. Obšč. 23 (1970); English transl., Trans. Moscow Math. Soc. 23 (1970), 1-35.

2. R. Ellis, Lectures on topological dynamics, Benjamin, New York, 1969. 
3. R. Ellis and R. Johnson, Topological dynamics and linear differential systems, J. Differential Equations (to appear).

4. A. Fathi and M. R. Herman, Existence de diffeomorphismes minimaux, Proc. Conf. Systèmes Dynamiques, Varsovie (1977), Astérisque 49, pp. 37-59.

5. H. Furstenberg, Strict ergodicity and transformations of the torus, Amer. J. Math. 83 (1961), 583-616.

6. S. Glasner and B. Weiss, On the construction of minimal skew-products, Israel J. Math. 34 (1979), 321-336.

7. R. Johnson, Almost periodic functions with unbounded integral, Pacific J. Math. 87 (1980), 347-362.

8. ___ Measurable subbundles in linear skew-product flows, Illinois J. Math. 23 (1979), 183-198.

9. Equations 37 (1980), 184-205.

10. V. V. Nemytskii and V. V. Stepanov, Qualitative theory of differential equations, English transl., Princeton Univ. Press, Princeton, N. J., 1960.

11. R. J. Sacker and G. R. Sell, A spectral theory for linear differential systems, J. Differential Equations 27 (1978), 320-358.

12. G. R. Sell, Linear differential systems, Lecture Notes, University of Minnesota, 1975.

13. W. A. Veech, Topological dynamics, Bull. Amer. Math. Soc. 83 (1977), 775-830.

Departiment of Mathematics, University of Minnesota, MinNeapolis, MinNesota 55455

Department of Mathematics, University of Southern Calmorni, los Angeles, Caltrornia 90007 (Current address) 